\title{
Dabigatran: how the drug company withheld important analyses
}

\author{
In an investigation by The BMJ Deborah Cohen finds that recommendations for use of new \\ generation oral anticoagulants may be flawed because regulators did not see evidence showing \\ that monitoring drug plasma levels could improve safety
}

\section{Deborah Cohen investigations editor, The BMJ}

An investigation by The BMJ shows how the manufacturers of a blockbuster anticoagulant stroke drug withheld from the regulators important analyses regarding how to use the drug as safely and effectively as possible.

Dabigatran is one of a new generation of oral anticoagulants for stroke prevention in patients with non-valvular atrial fibrillation recently recommended in guidelines from the National Institute for Health and Care Excellence for England and Wales. ${ }^{1}$ Guidelines in the US, Europe, and Canada have similarly recommended these drugs, in part because they don't require monitoring of plasma levels or anticoagulant activity and subsequent dose adjustment, unlike older treatments such as warfarin. ${ }^{2-4}$

Yet information about dabigatran disclosed through previously confidential internal company documents released during litigation in the US - which they have settled for $\$ 650 \mathrm{~m}(£ 380 \mathrm{~m}$; $€ 480 \mathrm{~m}$ ) - and as a result of an investigation by The BMJ, show that the evidence on which these guidelines were based is incomplete.

In fact, Boehringer Ingelheim, the maker of dabigatran, has failed to share with regulators information about the potential benefits of monitoring anticoagulant activity and adjusting the dose to make sure the drug is working as safely and effectively as possible. The company also withheld analyses that calculated how many major bleeds dose adjustment could prevent. The company says that this information was not shared because the analysis did not provide a reliable prediction of patient outcomes.

Anticoagulation is a risky procedure. In the UK, warfarin is one of the drugs most commonly implicated in emergency hospital admissions as a result of major bleeds ${ }^{5}$ and the drug's anticoagulant activity is monitored to reduce those risks.

In the single key trial comparing dabigatran with warfarin in non-valvular atrial fibrillation, major and minor bleeding occurred in $16.4 \%$ of patients a year taking the higher dose of dabigatran compared with $18.15 \%$ a year for warfarin. However, even after the regulators asked Boehringer to re-examine the trial data for missed events, it is still not clear whether we know how many fatal and life threatening bleeds there were in the trial. $^{6}$

Nevertheless, internal documents show how the company had produced extensive analyses that show how that bleeding risk may be reduced. The company found that if the plasma levels of the drug were measured and the dose was adjusted accordingly major bleeds could be reduced by $30-40 \%$ compared with well controlled warfarin. The adjustment would have little or no effect on the risk of ischaemic stroke. It has also identified the plasma levels at which the dose adjustment should occur to reduce the risk of a major bleed.

The conclusion of the analyses was: "Optimally used (=titrated) dabigatran has the potential to provide patients an even better efficacy and safety profile than fixed dose dabigatran and also a better safety and efficacy profile than a matched warfarin group."

But the company has told The BMJ that it has not shared this information with either doctors or regulators. The European Medicines Agency confirmed this, adding, "If we discover that the company withheld any relevant information, we will not hesitate to take necessary action" and will make "changes to the current recommendations."

Internal emails released during litigation perhaps show that some within the company did not want these conclusions to be known. During internal email discussions about the potential merits of drug plasma monitoring one Boehringer employee, whose name has been redacted, said: "This may not be a onetime test and could result in a more complex message (regular monitoring) and a weaker value proposition." Even as employees expressed concerns that elderly patients were being harmed, the company did not share these analyses with the regulators.

However, the company said that the emails made public had been selected to show it in a bad light and restated its claim that 
the anticoagulant activity or plasma concentrations of dabigatran do not need to be monitored.

"The analysis did not provide a reliable prediction of patient outcomes, and therefore we did not share the simulation with FDA or EMA," a spokesperson told The BMJ.

"Our scientists determined, and the FDA concurred, that the research does not support making dosage decisions based on plasma concentrations - a conclusion based solely on science and patient welfare," he said.

The spokesperson also reiterated the safety of dabigatran. "The FDA has publicly reported its own post-marketing analysis, concluding that dabigatran's post-marketing bleeding rates do not appear to be higher than [those with] warfarin," he said.

\section{Market advantage}

Crucially dabigatran was developed and marketed to be used in fixed dose regimens without the need for dose titration or monitoring of blood levels. This is considered to be a substantial advantage over warfarin.

At every stage in dabigatran's evaluation, licensing, and marketing, the claim that there was no need to monitor drug levels has been central. It has been a factor in the cost benefit evaluations by bodies like NICE and the successful marketing and widespread uptake of the drug.

It was even highlighted in an FDA press statement in 2010 at the time of its US approval:

"Unlike warfarin, which requires patients to undergo periodic monitoring with blood tests, such monitoring is not necessary for Pradaxa [dabigatran]," said Norman Stockbridge, director of the division of cardiovascular and renal products in the FDA's Center for Drug Evaluation and Research. ${ }^{7}$

The company's marketing messages to patients with atrial fibrillation in the US have also included this message prominently: "There are important differences between warfarin and Pradaxa," says its advertisement, one of which is that there is "No need for regular blood tests to see if your blood-thinning level is in the right range."

The fact that dabigatran was the first new oral anticoagulant for over half a century also allowed the new drug to benefit from regulatory policies in the US promoting innovation; before being licensed for atrial fibrillation it was studied in a single large phase III trial rather than in at least two trials, as is normally required for approval. ${ }^{9}$

Internal documents show that the trial was designed to "validate a NOAC [new oral anticoagulant] with no monitoring." The trial, called RE-LY, randomised participants to either warfarin or one of two doses of dabigatran $(150 \mathrm{mg}$ or $110 \mathrm{mg}$ twice daily), and was published in the New England Journal of Medicine in September 2009. ${ }^{10}$

The paper concluded that patients given the $150 \mathrm{mg}$ twice daily dose of dabigatran had lower rates of stroke or systemic embolism than those given warfarin. They also had similar rates of major bleeding.

However, the regulators had concerns about the design and oversight of the trial, which initially delayed the drug's approval. ${ }^{11}$ The FDA mandated a review of missed events after concerns about data quality. ${ }^{6}$

\section{Regulators' questions before licensing}

In the run-up to dabigatran being licensed, discussions about the possible need to monitor dabigatran came up at both the FDA and the EMA. Documents obtained under freedom of information show the EMA was concerned about the need to monitor the plasma levels of the drug to reduce the risk of bleeding; not just at the time of the decision to grant a licence to market the drug for stroke prevention in non-valvular atrial fibrillation in 2011, but also later when widespread use of the drug led to safety concerns.

They also show that the drug's licence in Europe was conditional on the availability of an accurate test to monitor the drug. This was decided to be the Hemoclot test. However, the agency repeatedly relied on the drug company for answers and to be transparent and open about the interpretation of their data. ${ }^{12}$

EMA documents from early 2010 also show that Boehringer had "identified dabigatran concentrations not to be exceeded because of the increased risk of bleeding ... The $200 \mathrm{ng} / \mathrm{mL}$ concentration is the value at trough not to be exceeded because of the increased risk of bleeding."

This value is reiterated in EMA's published drug assessment report. It also stipulates a lower end of the range of $48 \mathrm{ng} / \mathrm{mL} .^{13}$ During the US drug approval process in September 2010 one FDA adviser also raised the question of monitoring dabigatran because of the large differences in plasma levels among people taking the drug.

"I'm struck by what my eyeball tells me about a five-fold variability [in plasma levels] within the $90 \%$ confidence [interval] of the 150-dose. That seems awfully big to me in a drug that we're proposing to use without therapeutic monitoring," said Darren McGuire, a cardiologist on the panel in 2010. However, McGuire's concerns were not pursued by the agency.

So did Boehringer give this information to the FDA, as it had to the EMA? And if the EMA assessment included an upper threshold why isn't it included in prescribing information to UK doctors? A spokesperson for Boehringer said that the company "never told EMA or any regulatory authority that 200 ng/mL was a level "not to be exceeded,",

The company spokesperson said that EMA had agreed to say in the label that dabigatran concentrations greater than 200 $\mathrm{ng} / \mathrm{mL}$ "may be associated with an increased risk of bleeding," adding that as a result The BMJ's questions about whether this information has also been shared with the FDA and how many lives could have been saved if the upper limit of $200 \mathrm{ng} / \mathrm{mL}$ had been shared more widely was "moot."

However, internal documents show that the scientists the company had used to coordinate the trial show that EMA's statement might have merit. When discussing how best to publish analyses of data from the RE-LY trial, Stuart Connolly, one of the principal investigators of the RE-LY trial, said in an email in July 2012: "There is very good reason to never go above $200 \mathrm{ng} / \mathrm{ml}$. It is less clear at the low end due to the paucity of events but somewhere around $40-50$ seems prudent for a lower boundary."

In the end, the FDA approved dabigatran in October 2010 for use in stroke prevention in non-valvular atrial fibrillation without the need to adjust the dose in each patient; the EMA followed suit in August 2011.

Although the EMA approved the $110 \mathrm{mg}$ and $150 \mathrm{mg}$ doses, the FDA approved only the $150 \mathrm{mg}$ twice daily dose and asked for a $75 \mathrm{mg}$ twice daily dose to be developed for patients with severe renal impairment. ${ }^{12}$ The $75 \mathrm{mg}$ dose was not tested in clinical trials and was chosen on the basis of pharmacodynamic and pharmacokinetic data collected by the company. 


\section{What is dabigatran?}

Dabigatran is one of the new oral anticoagulants and is known as a direct thrombin inhibitor. Other new oral anticoagulant drugs are rivaroxaban and apixaban and are direct factor $\mathrm{Xa}$ inhibitors. Before this new group of drugs emerged, vitamin $\mathrm{K}$ antagonists, such as warfarin, were the mainstay of antithrombotic therapy. When dabigatran was licensed in Europe in 2008, it was the first new oral anticoagulant to be approved in over 50 years.

\section{Early concerns}

Once on the market, dabigatran proved a rapid success. By April 2012, it had achieved blockbuster status (where annual global turnover for a medicine exceeds $\$ 1 \mathrm{bn}$ ), prompting Boehringer board member Hubertus von Baumbach to say: "The launch of Pradaxa [dabigatran] is among the most successful market introductions in the pharmaceutical industry in the past few years."

But even as the sales of dabigatran were rapidly growing, concerns about fatal bleeds were beginning to emerge_-particularly in elderly people, who are at much higher risk of bleeding.

Indeed, during discussions about licensing the drug for non-valvular atrial fibrillation, the EMA was concerned that dabigatran would be "largely used in an elderly population which is known to be at a higher risk of bleeding" - a concern that internal company documents show to be justified.

Boehringer Ingelheim marketing data showed that " $45 \%$ of Pradaxa patients are 76 years or older" and " $30 \%$ of patients are 80 years or older." However, only $40 \%$ of participants in the RE-LY trial were over 75 and $17 \%$ over 80 .

By the end of 2011 regulators were concerned as postmarket reports accumulated about cases of severe bleeding and deaths among patients taking dabigatran. A QuarterWatch report analysed all the adverse events submitted to the FDA's reporting system in $2011 .{ }^{14}$ It found the most commonly identified drugs reported to the FDA were the anticoagulants dabigatran and warfarin. For dabigatran alone, this included 542 patient deaths and 2367 reports of haemorrhage. Warfarin accounted for 72 deaths in the same period.

"The need to achieve greater safety in anticoagulant treatment should be a drug safety priority. While dabigatran is replacing warfarin in the market as a drug that is easier to use, the priority need is for stroke prevention treatments that are safer," the report authors wrote. ${ }^{14}$

\section{What did Boehringer know?}

Documents released during US litigation have shown that the company worried about how to improve the drug's safety. Questions were being asked about whether the drug had a therapeutic range within which risks could be minimised and benefits could be maximised and if it would therefore need monitoring and dose adjustment.

Although the RE-LY trial protocol did not require monitoring of blood levels in patients taking dabigatran, the investigators collected drug plasma concentrations during the trial. Internal documents circulated within the company in August 2011 show that employees completed a subgroup analysis of these data.

Some of the conclusions of this analysis were eventually published online in the Journal of the American College of Cardiology in September 2013 under the title "The effect of dabigatran plasma concentrations and patient characteristics on the frequency of ischemic stroke and major bleeding in atrial fibrillation patients." ${ }^{15}$ The lead author was Paul Reilly, a Boehringer employee, and coauthors included other company employees as well as Stuart Connolly and Salim Yusef from
McMaster University in Canada and Lars Wallentin from the Uppsala Clinical Research Centre, Sweden.

The paper, which was first drafted in August 2011, examined a critical question: how much did the benefits (reduced risk of ischaemic stroke) and harms (increased risk of bleeding) vary across the drug's plasma concentration range. This "has important implications for the benefit-risk ratio in individual patients," both the draft and published papers said. They found that there was a fivefold variation in blood plasma concentration with each dose.

Reilly's paper reported that renal function was the most important determinant of dabigatran concentration, and age is the most important covariate. "The large majority of patients achieve a favourable balance of benefit and risk with a fixed dose of DE [dabigatran] 110 or DE [dabigatran] 150, guided by a consideration of patient characteristics," the published paper said.

However, the 2011 draft also suggested there was an optimal plasma concentration range of the drug and went beyond tailoring dose according to patient characteristics.

It found that there was a fivefold variation in blood plasma concentration with each dose. "Monitoring of plasma concentrations or antithrombotic activity ... would be required to identify these patients. A dose adjustment could improve the benefit-risk ratio," according to the 2011 draft seen by The BMJ.

But internal emails released during US litigation show how the company grappled with the implications of the paper. In an internal email in 2011, Andreas Clemens, a medical team leader for the drug, stated that he was "phobic" and "not happy with the conclusion"- that an optimal balance between benefit and risk occurs in the range of concentrations between $40 \mathrm{ng} / \mathrm{mL}$ and $215 \mathrm{ng} / \mathrm{mL}$.

The company knew what damage the paper might do-and yet emails show that in July 2012 Connolly thought that it was a good paper that "will have an impact on thinking about dabigatran" and points to "optimization of safety and efficacy in the range from $40-200 \mathrm{ng} / \mathrm{mL}$."

However, the scientists' concern conflicted with that of some in the company. An email in October 2012 shows a company official saying that "The publication will [do] more harm than be useful for us, neither in the market but especially harmful in the discussions in the regulatory bodies."

Clemens also wrote that he believed that the findings were important and should be published but with revision. "The world is crying for this information-but the tricky part is that we have to tailor the messages smart."

Emails from February 2013 show that company employee Jutta Heinrich-Nols wrote to other employees to recommend that the company reconsider whether to publish this study.

"This will make any defense of no monitoring to HA [health authorities] extremely difficult (i.e. Health Canada, TGA) and undermine our efforts to compete with other NOACs [new oral anticoagulants]. As I am not empowered to release or stop any publications I would like to ask you to check once again whether this is really wanted.," an email said. 
Publishing the research results, she warned, could make it "extremely difficult" for the company to defend its long-held position to regulators that dabigatran did not require monitoring.

The BMJ contacted the three independent doctors working with the data and asked them about the apparent conflict around what was contained in the paper.

Wallentin said that while the academics had access to the RE-LY database and freedom to analyse what they wanted, "all scientific projects are submitted to and approved by the RE-LY publications committee. The content of all publications are of course at the end based on discussions followed by modifications to reach a consensus among all coauthors," he said.

The BMJ has found that the company did defend the notion that the drug did not require monitoring of plasma levels or anticoagulant activity to health authorities.

As the number of fatal bleeds accumulated and company employees deliberated Reilly's analyses, the EMA started to formally consider the key issue that the manufacturer was trying to avoid: that dabigatran would need to be monitored and the dose adjusted. In early 2012 the agency convened its scientific advisory group of experts to give it advice about these issues. Specifically, it wanted to know if there was a "need for stronger and more specific recommendations for measurement of dabigatran related anticoagulation," specifically in those groups at increased risk of bleeding.

The European agency also asked the committee to "discuss and suggest appropriate monitoring frequency and laboratory tests." On 9 March, 2012, Boehringer gave a presentation to the committee, details of which The BMJ has obtained under a freedom of information request, along with the agency's minutes of the meeting.

The EMA's minutes show that routine monitoring of anticoagulant activity was discussed "in depth" by the committee. However, most experts voted against it.

The committee thought that this was justified because "the desired plasma drug level and the therapeutic window are not known" and "there is significant variability of more widely available tests such as the aPTT [activated partial thromboplastin time] making interpretation difficult."

In the end, the final recommendations simply stressed the need to monitor renal function and patient characteristics before and during treatment and "make dose reductions in certain patients"- and not routinely measure plasma concentrations or anticoagulant activity. Dabigatran is predominantly excreted by the kidneys.

Some of the analyses and conclusions outlined in Reilly's 2011 paper, which was produced over six months before EMA's safety meeting in March 2012, were absent from the company's presentation to the committee.

Aspects of Paul Reilly's 2011 paper that were not in the company's presentation include a graph showing that beyond a certain plasma concentration of the drug major bleeding events continued to increase as the plasma levels increased with little effect on rates of stroke and systemic embolism. ${ }^{12}$ This graph was, however, published in 2013 in the Journal of the American College of Cardiology. Also absent from the presentation were data showing that some people taking dabigatran may have a suboptimal dose, putting them at "an appreciably higher" stroke risk.

In the meeting, company officials highlighted the importance of measuring creatinine clearance to assess renal function. The company also chose to present statistics in which the plasma level variability seemed to be about 2.3-fold instead of 5.5-fold as documented in Reilly's paper. The presentation added that the European label currently includes monitoring for renal function and cut-off values for dabigatran exposures with increased bleeding risk and plasma level data from RE-LY. But stated: "Routine monitoring of the anticoagulant activity is not necessary."

The unpublished version of the Reilly paper, however, stated that "targeting a specific concentration range may optimize the benefit-risk ... A dose adjustment could improve benefit-risk ratio," it said. But the company's presentation to the EMA's ad-hoc committee did not include this information.

The BMJ asked Boehringer if it was confident that it had presented all the internal analyses of the RE-LY trial data that were available to them to EMA's ad-hoc safety meeting for the assembled experts to discuss.

We also asked if it had made the European agency aware during the meeting that company analyses suggested "targeting a specific concentration range may optimize the benefit-risk" and that: "monitoring of plasma concentrations or antithrombotic activity ... would be required to identify these patients. A dose adjustment could improve the benefit-risk ratio," as had been described in the draft publication.

A spokesperson for the company said: "At the meeting, BI presented analyses and information that were relevant to these issues and available at the time. All RE-LY trial data, along with extensive analyses of that data, had previously been provided to EMA."

The spokesperson also said that the company did not tell EMA's safety committee that monitoring plasma concentrations and targeting a specific concentration range were being discussed internally as they were "hypotheses in drafts of a paper that the authors of that paper rejected as they refined their analysis."

The company spokesperson also stated that such models "are inherently imprecise and carry a margin of error." The reasons for this, the spokesperson said, is that the modelling is "not based on data that directly measured the efficacy and safety outcomes for patients subject to monitoring and dose adjustment," adding that: "the data that the simulation was based on was not sufficiently robust to reliably derive plasma concentration thresholds for dose adjustment and then predict patient outcomes."

The BMJ asked Boehringer if it could provide any more information to clarify the issue and to support its assertion that the modelling wasn't reliable. It's not clear from the information provided when or why these particular models were rejected and considered to be inaccurate given that the data and the models have been used for other purposes, including publications and regulatory decisions.

The company has said that it provided all the trial data—which it is obliged to do-and extensive analyses. However, the question of the extent of sharing of internal company analyses of the data with the regulator is a grey area, as there is no legal obligation on a company to share such data.

However, Steve Nissen, department chair of cardiovascular medicine at the Cleveland Clinic and one of the members of the FDA's advisory committee considering dabigatran for use in non-valvular atrial fibrillation, told The BMJ: "If there is clinically useful information about the relationship between drug levels and the safety of dabigatran, it is the moral obligation of the company and its investigators to share this information with the medical community. Withholding such information for commercial purposes is unacceptable." 


\section{The Hemoclot test}

It wasn't just the absence of a therapeutic range that persuaded the ad-hoc meeting to stop short of recommending the measurement of the drug's plasma levels. There was the problem of how to test the dabigatran's anticoagulant activity or its plasma levels.

Before dabigatran was licensed by EMA, documents obtained under freedom of information show that the European agency insisted on there being a device to monitor the drug levels available on the market. It was decided that this should be the Hemoclot test (diluted thrombin time).

What tests to use was discussed at the 2012 meeting. The company's presentation showed a graph using clotting time plotted against plasma concentration. But this was dismissed as having "limited sensitivity."

The Hemoclot test was discussed at the 2012 meeting - and proposed by some experts-but rather than discuss ways of ensuring its uptake, the test was dismissed by the committee as it is "under development" and "may not be available in many laboratories in the EU and some time is required to obtain the results." (In the US the Hemoclot thrombin inhibitor assay is available only for research use.)

Why the committee came to that conclusion rather than suggest wider use is unclear. That same month (March 2012) company employees published a paper that highlighted the accuracy of the Hemoclot test in patients taking dabigatran.

The paper stated that Hemoclot thrombin inhibitor assay was a "rapid, established, standardized and calibrated" and "should provide accurate and consistent results" when assessing both the anticoagulant activity and calculating plasma concentrations of dabigatran. ${ }^{16}$

\section{Commercial interests}

From an early stage, Boehringer planned to develop and market a drug that did not require plasma level monitoring. Internal documents show that even though there had been deaths associated with major bleeds in the clinical trial and there was no antidote- a decision had been made not to support the development of a bedside monitoring device.

The rationale for this was laid bare in an email on 3 June 2010. An employee from the cardiology division of the company brought up the issue of the utility of such a device. In an email they said: " 2 years ago [in 2008] there was an informed decision NOT to develop this. As this would go against the "no monitoring' idea/claim."

Soon after Boehringer told the EMA's committee that adjusting dose for plasma levels was not necessary, it was considering relaunching the drug based on adjusting the dose to a specific therapeutic range. The company wanted to know if it could find a "unique selling" point for dabigatran since rivaroxaban and apixaban - two other new oral anticoagulants-had come onto the market. "Could individualised dosing be a unique selling point for Pradaxa in the marketplace," a June 2012 document entitled "Potential mid to long term strategy for Pradaxa in SPAF [stroke prevention in atrial fibrillation]" said.

The document noted that prescribers often wanted to know the extent of the anticoagulation each patient is receiving with their current anticoagulant. Company employees produced yet more analyses, which they summed up in the strategy document and accompanying slide presentation circulated to Boehringer executives.

It analysed whether "a one-time initial measurement (perhaps repeated annually and in some instances, such as moderate renal impairment, in shorter intervals)" followed by titration of dabigitran to reach an optimal dose would be the safest and most effective way of using the drug.

After an "intense effort" using data simulations and data from RE-LY, it found that by doing this, it "could preserve the effect on ischemic stroke prevention but with a reduction of major bleeding events compared to well controlled warfarin of perhaps up to 30-40\%." The data also suggested that such an approach would even lead to fewer gastrointestinal bleeds with dabigatran "compared to warfarin in such a setting."

The company were also keen to get the $110 \mathrm{mg}$ twice daily dose on the market in the US, and this would be needed for individualised dosing. ${ }^{12}$

"[The] FDA has indicated that such modeling data, together with clinical data (eg, a PK/PD

[pharmacokinetic/pharmacodynamic] study) on a titration strategy, may be the only way forward to an approval of the 110 mg dose in the US," the document said. After considering regulatory and other obstacles, the company has thus far not elected to pursue this strategy.

By comparing a $150 \mathrm{mg}$ twice daily dose used under recommendations for use stipulated by the EMA—which advocates patient selection and renal monitoring - to a regimen in which dabigatran that was monitored and titrated accordingly, the company calculated that major bleeds could also be reduced.

The presentation also outlined proposed dose adjustment according to specified plasma levels, which formed the basis of their analysis (figure $\downarrow$ ).

But neither doctors nor the regulators have ever been made aware of the company calculations circulated in June 2012 - including the number of major bleeds that could be prevented and at what plasma levels the drug should be titrated.

A Boehringer spokesperson said: "The analysis did not provide a reliable prediction of patient outcomes, and therefore we did not share the simulation with FDA or EMA," adding: "All of the data that was used for the analysis had already been provided to the regulatory authorities"

But in their mid to long term strategy document, company officials also wondered if patients who had low levels of dabigatran in their blood despite receiving the higher dose would have to stop treatment. And if so, what percentage of people with atrial fibrillation would this account for?

There were also questions over what the implications would mean for people taking dabigatran for conditions other than atrial fibrillation (such as venous thromboembolism). "Will this negatively impact the product perception?" the document said.

However, detailed information about the optimal therapeutic range would have been useful to doctors. Paul Chin, a clinical pharmacologist, at Christchurch Hospital, University of Otago, in New Zealand, has been trying to identify the optimal range for patients for some time using what information was publicly available. "It would have been useful for doctors to know that patients at the extremes of trough concentrations have much higher risks of stroke (very low concentrations) or bleeding (very high concentrations), and how to detect this," he said.

Chin says that he asked Boehringer for RE-LY data on plasma concentrations and glomerular filtration rate back in 2011. "But we got what is probably the standard reply that it's confidential and would have to be taken up with corporate and we'll get back to you sometime," he said.

In June 2012, he and a group of other academics published a paper in the British Journal of Clinical Pharmacology that caught the eye of company executives. It stated that testing in clinical practice has been "largely downplayed." 
The paper pointed out that the UK guidelines from Boehringer advocating renal testing in some patients did not go far enough, "leaving some individuals likely to be overdosed and some perhaps underdosed." It advocated the use of the Hemoclot test. Internally, the paper triggered discussions. One employee was worried about the adverse effects of dabigatran-particularly in older people. "We should not ignore that in those over 80 years old, even the $110 \mathrm{mg}$ had a unfavourable trend compared to warfarin (major bleeding 80 years: hazard ratio 1.12 (95\% confidence interval $0.84,1.49)$," the employee wrote, arguing that Boehringer needed to study this further before another independent group did.

Facing concerns that the company had not put the data contained within the Reilly paper into the public domain earlier, Reilly and colleagues stated in a letter to the Journal of the American College of Cardiology: "Of the new oral anticoagulants, RE-LY is the only trial that has published extensive data on this topic. It is likely that other anticoagulants will also exhibit variability in blood concentrations."

Boehringer's Reilly makes a point worth pursuing. Rivaroxaban and apixaban were also marketed on the theme that plasma level dose adjustment was not needed, as it is with warfarin. More systematic and independent study is needed to establish what price, in terms of preventable haemorrhage and death, is being paid for each of the new drugs in the name of ease of use.

Indeed, Hugo ten Cate, medical director of the Maastricht thrombosis anticoagulation clinic and coeditor in chief of Thrombosis Journal, has been concerned about the lack of published studies on dose adjustment in the new oral anticoagulants for some time.

This combined with a lack of antidote has been a "major hurdle in the safe introduction of NOACs," he wrote in March 2012.

"It is critical that pharmaceutical companies take their responsibilities and provide and publish all relevant data on drug levels and coagulation test responses so that it becomes clear what the approximate therapeutic and harmful ranges of laboratory test outcomes are, for each anticoagulant agent. There is no good reason not to be transparent in these matters, even if it would entail the small risk that doctors would want to optimise therapy based on lab test results," he said.
Competing interests: I have read and understood BMJ policy on declaration of interests and have no relevant interests to declare.

Provenance and peer review: Commissioned; externally peer reviewed.

1 NICE Implementation Collaborative. Consensus. Supporting local implementation of NICE guidance on use of the non-Vitamin K antagonist oral anticoagulants (NOACs) in non-valvular atrial fibrillation. www.nice.org.uk/resource/CG180/pdf/c/cg180-atrialfibrillation-nic-consensus-statement-on-the-use-of-noacs?id=gvyb3hjdqrcjtn6ytpwx3ydb64.

2 Camm AJ, Lip GY, De Caterina R, Savelieva I, Atar D, Hohnloser SH, et al. 2012 focused update of the ESC Guidelines for the management of atrial fibrillation. Eur Heart $J$ 2012;33:2719-47.

$3 \mathrm{AHA} / \mathrm{ACC} / \mathrm{HRS}$ guideline for the management of patients with atrial fibrillation: a report of the American College of Cardiology/American Heart Association Task Force on Practice Guidelines and the Heart Rhythm Society. J Am Coll Cardiol 2014 Mar 28. [Epub ahead of print.]

4 Skanes AC, Healey JS, Cairns JA, Dorian P, Gillis AM, McMurtry MS, et al. Focused 2012 update of the Canadian Cardiovascular Society atrial fibrillation guidelines: recommendations for stroke prevention and rate/rhythm control. Can J Cardiol 2012;28:125-36.

5 Pirmohamed M, James S, Meakin S, Green C, Scott AK, Walley TJ, et al. Adverse drug reactions as cause of admission to hospital: prospective analysis of 18820 patients. BMJ 2004;329:15-9.

6 Cohen D. Concerns over data in key dabigatran trial. BMJ 2014;349:g4747.

7 FDA. FDA approves Pradaxa to prevent stroke in people with atrial fibrillation. Press release, 16 October 2010. www.fda.gov/newsevents/newsroom/pressannouncements/ ucm230241.htm.

8 Staton T. Pradaxa hits blockbuster mark, swelling Boehringer sales. FiercePharma 2012 Mar 24. www.fiercepharma.com/story/pradaxa-hits-blockbuster-mark-swelling-boehringersales/2012-04-24.

9 Moore TJ, Furberg TC. The safety risks of innovation: the FDA's expedited drug development pathway. JAMA 2012;308:869-70.

10 Connolly SJ, Ezekowitz MD, Yusuf S, Eikelboom J, Oldgren J, Parekh A, et al. Dabigatran versus warfarin in patients with atrial fibrillation. N Engl J Med 2009;361:1139-51.

11 Heneghan C, Onakpoya I, Mahtani KR. Questions over dabigatran's key trial. BMJ 2014;349:g4560.

12 Moore TJ, Cohen MR, Mattison DR. Dabigatran, bleeding, and the regulators. BMJ 2014;349: g4517.

13 Committee for Medicinal Products for Human Use (CHMP), Pradaxa assessment report, 9 June 2011, EMA/CHMP/203468/2011.

14 Moore TJ, Cohen MR, Furberg CD. Anticoagulants the leading reported drug risk in 2012. QuarterWatch 2012 Quarter 4. www.ismp.org/quarterwatch/pdfs/2011Q4.pdf.

15 Reilly PA, Lehr T, Haertter S, Connolly SJ, Yusuf S, Eikelboom JW, et al. The effect of dabigatran plasma concentrations and patient characteristics on the frequency of ischemic stroke and major bleeding in atrial fibrillation patients. J Am Coll Cardiol 2014;63:321-8.

16 Stangier J, Feuring M. Using the HEMOCLOT direct thrombin inhibitor assay to determine plasma concentrations of dabigatran. Blood Coagul Fibrinolysis 2012;23:138-43.

17 Reilly PA, Connolly SJ, Yusuf S, Eikelboom JW, Ezekowitz MD, Wallentin L. Reply to Letter to the Editor: Regarding "The effect of dabigatran plasma concentrations ...." J Am Coll Cardiol 2014, doi:10.1016/j.jacc.2014.04.012.

Cite this as: BMJ 2014;349:g4670

๑ BMJ Publishing Group Ltd 2014 


\section{Figure}

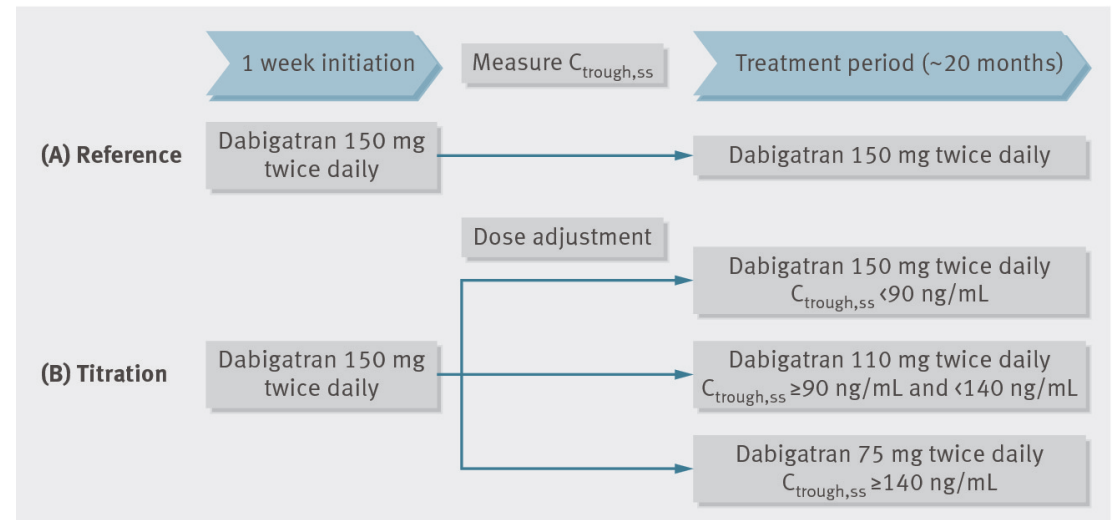

Boehringer's proposed dose adjustments for dabigatran 